\title{
WIDE-BANDGAP CIAS THIN-FILM PHOTOVOLTAICS WITH TRANSPARENT BACK CONTACTS FOR NEXT GENERATION SINGLE AND MULTI-JUNCTION DEVICES
}

\author{
Lawrence M. Woods, Ajay Kalla, Damian Gonzalez and Rosine Ribelin \\ ITN Energy Systems Inc., Littleton, CO, 80127
}

\section{INTRODUCTION}

Future spacecraft and high-altitude airship (HAA) technologies will require high array specific power (W/kg), which can be met using thin-film photovoltaics (PV) on lightweight and flexible substrates.(1) It has been calculated that the thin-film array technology, including the array support structure, begins to exceed the specific power of crystalline multi-junction arrays when the thin-film device efficiencies begin to exceed $12 \%$.(2) Thin-film PV devices have other advantages in that they are more easily integrated into HAA's, and are projected to be much less costly than their crystalline PV counterparts. Furthermore, it is likely that only thin-film array technology will be able to meet device specific power requirements exceeding $1 \mathrm{~kW} / \mathrm{kg}$ (photovoltaic and integrated substrate/blanket mass only). Of the various thin-film technologies, single junction and radiation resistant $\mathrm{CulnSe}_{2}$ (CIS) and associated alloys with gallium, aluminum and sulfur have achieved the highest levels of thin-film device performance, with the best efficiency, reaching 19.2\% under AM1.5 illumination conditions and on thick glass substrates.(3) Thus, it is anticipated that single- and tandem-junction devices with flexible substrates and based on CIS and related alloys could achieve the highest levels of thin-film space and HAA solar array performance.

ITN Energy Systems, Inc. (ITN) intends to meet the requirements for future high-altitude airship and spacecraft power by developing highly efficient single-junction and two-terminal monolithic tandem (multi-junction) PV cells using low-cost thin-films on lightweight flexible substrates. ITN will achieve this goal using thin-films of highefficiency and radiation resistant CIS partnered with bandgap-tunable CIS-alloys with Ga (CIGS) and/or Al (CIAS) on novel lightweight and flexible substrates. ITN is currently developing a widebandgap CIAS top cell on infra-red transparent back contacts and polyimide substrates to increase the single-junction performance over existing thin-film CIGS on thin metal foil. An illustration of this configuration is given in Figure 1. As a single-junction material, a device using a wide-bandgap CIAS solar absorber offers several performance advantages over standard lowbandgap CIGS devices: it is better tuned to the optimum bandgap for the AM0 solar spectrum; it is better suited for high voltage applications; it undergoes lower resistive losses for cells and modules; it transmits more unused infra-red light for lower temperature operation; and it produces higher power during high-temperature applications due to lower temperature coefficients. A widebandgap single junction with transparent back contacts also enables potential backside power generation and is applicable as the top cell and 
transparent interconnect components in a monolithic tandem device. This paper discusses the current performance, testing, and problems of the wide-bandgap single junction CIAS device with transparent back contacts.

\section{WIDE-BANDGAP CIAS ALLOY THIN-FILM DEVICES}

The electronic quality of the Culn ${ }_{1-x} \mathrm{Ga}_{x} \mathrm{Se}_{2}$ (CIGS) material has been shown to degrade for $\mathrm{x}>0.3$, thus the achievement of high-efficiency wide-bandgap CIGS devices is not as easy as simply increasing the Ga alloying in the CIGS material. The best CGS devices ( $\mathrm{x}=1$ or bandgap equal to $1.67 \mathrm{eV}$ ) were recently fabricated and tested at $9.53 \%$ under AM1.5 illumination (Reference: NREL report, "Polycrystalline Thin Film High Performance: NREL In-house Activities," March/April 2003 bimonthly); a good result but far from the $19.2 \%$ efficiency of the lowbandgap material. One approach to potentially overcome the bandgap limitations of CIGS devices is to alloy the CIS with aluminum instead of gallium, thus forming thin-film Culn ${ }_{1-x} \mathrm{Al}_{x} \mathrm{Se}_{2}(\mathrm{CIAS})$. This approach for thin-film photovoltaic devices was initiated by ITN in conjunction with the Institute of Energy Conversion (IEC) nearly four years ago, and is currently under continued development at ITN for large-area (production-like) CIAS depositions. The potential advantage of CIAS is that, for a given wide bandgap, much less Al alloying is needed compared to alloying with $\mathrm{Ga},(4)$ and thus potentially avoiding defect formation associated with higher alloy (Ga or Al) content materials. $(5,6)$ Indeed, recent information about CIAS deep defect density has indicated that Al alloying has more of an effect on the band tail defect distribution, rather than deep defects (Reference: Heath et al., Spring MRS 2003 , to be published). R\&D CIAS devices with efficiencies of about $11 \%$ have been fabricated with bandgap • $1.5 \mathrm{eV}(\mathrm{x}=0.5)$. Furthermore, excellent devices (eff. $\approx 17 \%$ under AM1.5) have recently been achieved using small amounts of $\mathrm{Al}$ alloying,(7) and with low substrate temperatures (450 ${ }^{\circ} \mathrm{C}$ ) during CIAS co-evaporated deposition.(8) This good performance capability for this material system at the lower substrate temperatures is important for the temperature-limited polyimide substrates and for reducing the stress to the bottom cells in monolithic tandem devices. For CIAS with more top-cell-optimal bandgaps of around $1.65 \mathrm{eV}$, the best devices to date are $7.8 \%$, but without a performance enhancing anti-reflective top coat, and is thus similar to the CGS performance mentioned above.(8) All these references to CIAS device performance were measured on devices with CIAS deposited without any bandgap tailoring vs. depth, which is typically associated with the highest performance devices. More latitude with bandgap tailoring is available for the wide-bandgap materials using the CIAS alloy system, given its endpoint bandgap of $2.7 \mathrm{eV}$ (CAS), compared to $1.68 \mathrm{eV}$ for CGS in the CIGS alloy system. ITN's CIAS device performance, from the large area and moving substrate deposition system (production like), show similar performance trends with Al content as stated above, and are currently achieving about $2 / 3^{\text {rds }}$ the efficiency of the best laboratory (small area, stationary substrate) devices. Some light IV curves from a series of CIAS devices with variable Al-content made using the large-area, moving substrate system at ITN are shown in the left side of Figure 2.
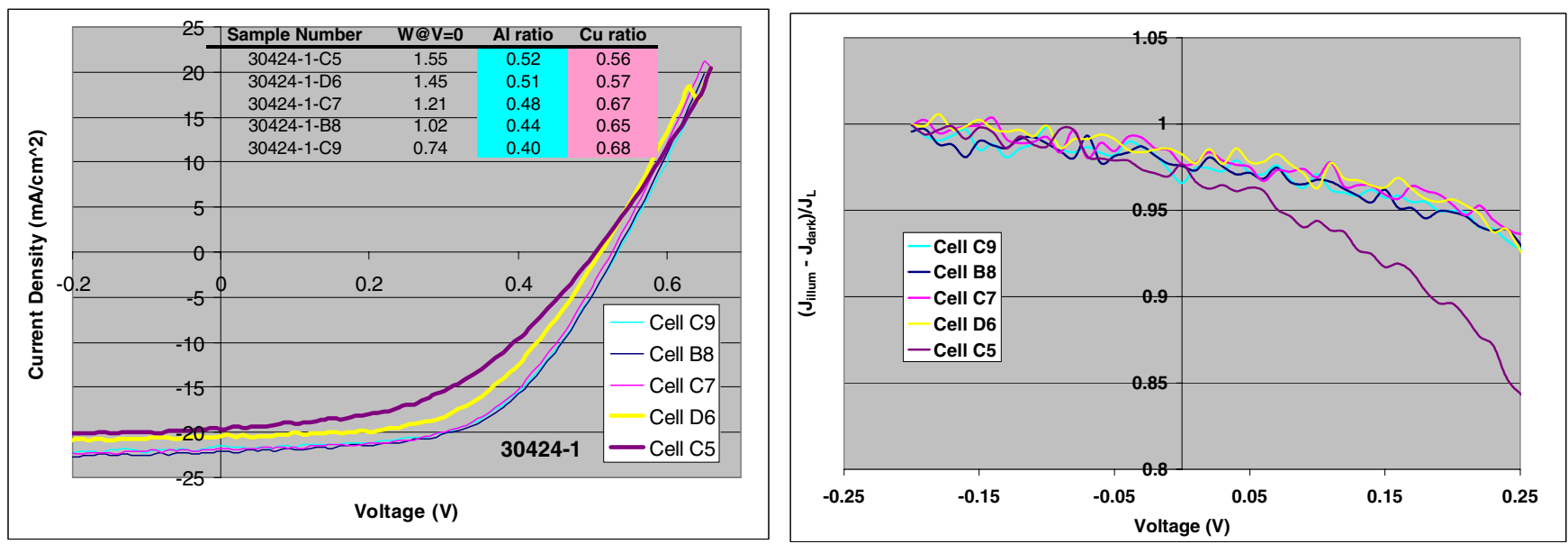

Figure 2 -Light IV curves (left) and voltage dependence of the normalized current difference (right) of small-area $\left(1 \mathrm{~cm}^{2}\right)$ wide-bandgap CIAS devices from the large-area, moving substrate deposition system. Device composition and zero bias depletion width $(\mathrm{W} @ \mathrm{~V}=0)$ are given in the inset tables. 
Table 1 - AM1.5 Light IV parameters for small-area $\left(1 \mathrm{~cm}^{2}\right)$ wide-bandgap CIAS devices shown in Figure 2.

\begin{tabular}{cccccccc} 
Device & Eff. (\%) & Voc (V) & Jsc $\left(\mathbf{m A} / \mathbf{c m}^{2}\right)$ & Fill Factor & Rs (ohms) & Rsh (ohm-cm) & Diode Factor \\
\hline $30424-1-C 5$ & 4.66 & 0.504 & 19.71 & 0.47 & 4.01 & 227.00 & 4.36 \\
$30424-1-D 6$ & 5.65 & 0.511 & 20.45 & 0.54 & 3.22 & 255.00 & 3.68 \\
$30424-1-C 7$ & 6.41 & 0.526 & 21.81 & 0.56 & 3.34 & 166.00 & 3.07 \\
$30424-1-B 8$ & 6.61 & 0.532 & 22.15 & 0.56 & 3.33 & 107.00 & 3.14 \\
$30424-1-C 9$ & 6.47 & 0.535 & 21.51 & 0.56 & 3.55 & 174.00 & 3.05
\end{tabular}

The IV curves indicate an apparent high series resistance problem that becomes worse when the Al-ratio exceeds 0.5. Closer examination shows that the series resistance (Rs) as determined using the intercept of a plot of $\mathrm{dV} / \mathrm{dJ}$ vs $\mathrm{J}^{-1}$, given in Table 1, does not change much with Al content. Furthermore, a plot with the voltage dependence of the normalized current difference (right side of Figure 2) shows significant voltage dependence in all devices, especially in the device with the highest Al-content. Thus, the IV curve features are partially a result of voltagedependent current collection. In addition, there is a decrease in the open-circuit voltage (Voc) for these higher Al content devices, instead of steadily increasing with increasing Al content. This is accompanied by an increased diode factor (A, in Table 1) and decreasing FF with increasing Al-content. Using the standard solar cell model, the series of measured light IV results can only be fitted by increasing the diode saturation current and diode factor with increasing Al-content, and thus indicates changes in the CIAS electronic properties with Al-content. Indeed, the zero bias depletion widths from Capacitance-Voltage (CV) testing, shown in the figure inset (in units of microns), indicate an increase in the junction depletion width with increasing Al-content. This indicates a decrease in the net doping density near the junction with increasing Al-content. Additional data on the zero bias depletion depth versus CIAS composition are shown in Figure 3, and confirm the correlation with Al content. In this figure the data is color-coded for different $\mathrm{Cu}$-ratio ranges, so that the effect of $\mathrm{Cu}$-ratio on the depletion width can be separated out. For this analysis, the depletion widths were calculated using a composition-dependent dielectric constant, $\varepsilon_{r}(\mathrm{x})$, which was estimated as linearly decreasing with Al-content between $\varepsilon_{\mathrm{r}}(0)=11.5$ and $\varepsilon_{\rho}(1)=8.8$, representing the respective CIS and CAS endpoint ternaries. This adjustment works to counteract the apparent trend, thus without it, the depletion width dependence on Al-content would have shown a higher correlation. Similarly, a reduction in depletion width from Cu-ratios around 0.63 is also apparent as shown in Figure 3 , but is not as strongly correlated as the Al-ratio. Here also the data is color coded for different Al-ratio ranges, so that the effect of Al-ratio on the depletion width can be separated out.
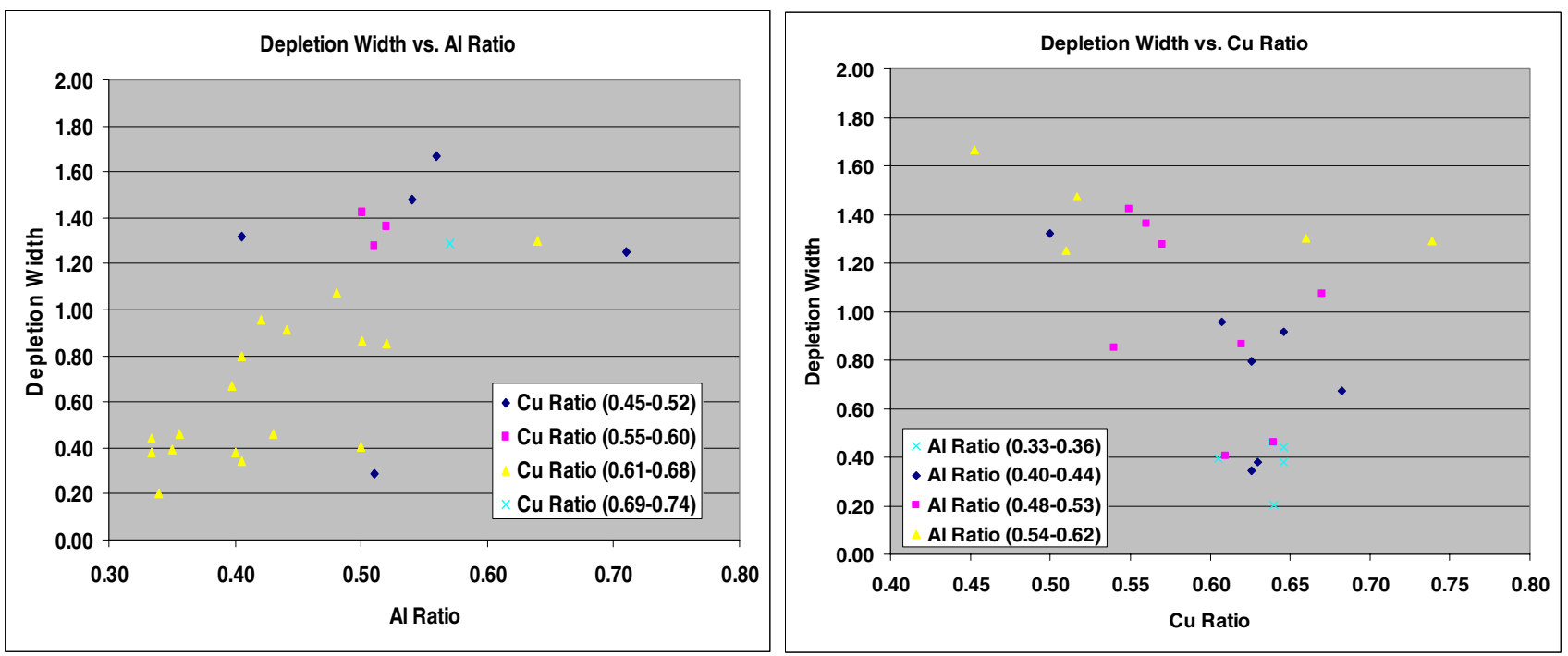

Figure 3 - Zero Bias depletion width versus Al-ratio (left) and Cu-ratio (right) for several devices. Data is adjusted for linearly vary dielectric constant with Al-content and color coded to bracket certain Cu-ratio ranges (left) and Al-ratio ranges (right). 
Owing to the lower net doping level in the junction region, the high Al and low Cu content (highly compensated) CIAS devices may have a more severe surface inversion (n-type) layer, a feature now commonly associated with low bandgap CIGS devices. This inversion layer, with an electron barrier at the CdS/inversion layer interface, could lead to the observed voltage dependent current collection and the kink feature sometimes observed in IV curves. A barrier in the conduction band at the CdS/inversion layer interface is possible with increasing conduction band maximum in the CIAS and an n-type inversion layer. This possibility is supported by the high sensitivity of the voltage dependent current collection to air annealing (surface sensitivity), which may reduce the inversion layer thickness and doping in high Al content CIAS devices. An IV curve example of this sensitivity is shown in Figure 4.

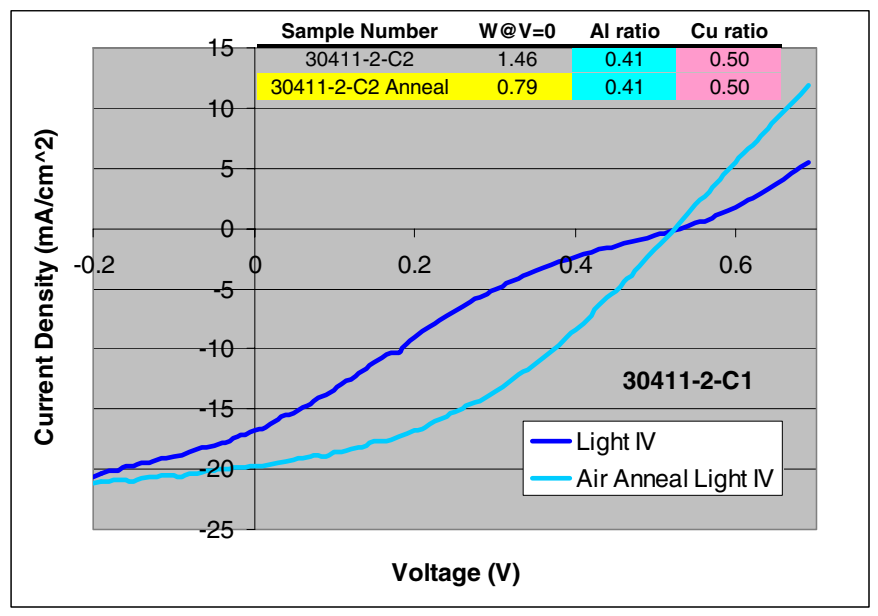

Figure 4 - Example IV's showing the effect of air anneal on high bandgap CIAS devices.

As expected, the short-circuit current (Jsc) decreases with increasing Al-content (bandgap). The quantum efficiency (QE) measurements shown in Figure 5 confirm the increasing bandgap with Al content, as indicated by the decreasing long wavelength cut-off with increasing Al content (left side of Figure 5). The QE measurements also indicate wavelength dependent collection with reduced collection at longer wavelengths and significant subbandgap collection. These characteristics worsen with increasing bandgap (Al-content), as does the maximum $\mathrm{QE}$, and is easier to see when the QE curves are aligned near their long-wavelength inflection points as shown in the right side of Figure 5.
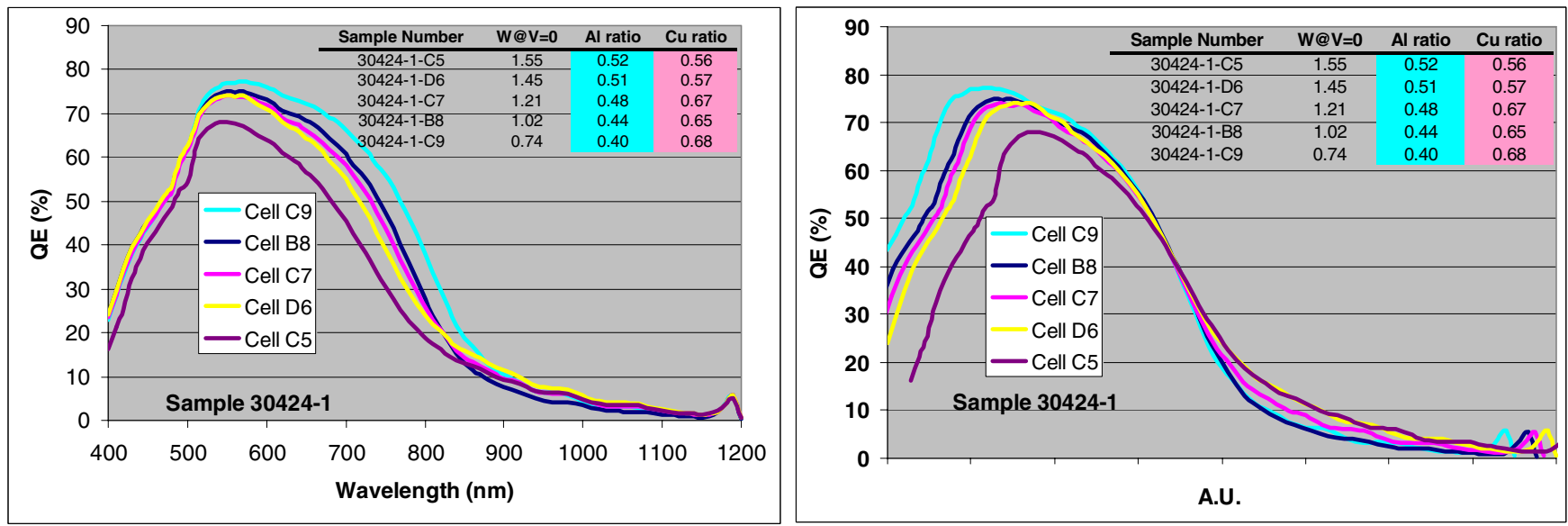

Figure 5 - QE's (right) of small-area $\left(1 \mathrm{~cm}^{2}\right)$ wide-bandgap CIAS devices from the large-area, moving substrate deposition system. Device composition and zero bias depletion width $(W @ V=0)$ are given in the inset tables. QE's on left are the same devices but adjusted to line-up the long wavelength inflection point. 
Devices with a depletion width extending almost completely through the CIAS thickness (higher Al content) do not give the expected better long wavelength collection efficiency, and indicate an increase in junction and bulk recombination.

\section{TRANSPARENT BACK CONTACTS}

Reverse bias capacitance-voltage profiling (not shown) of CdS/CIAS devices with standard opaque Mo back contacts, reveals that the net bulk doping density is decreasing with increasing Al-content and correlates with the zero bias depletion widths discussed above. The lowest values are measured to be in the low $10^{-15} \mathrm{~cm}^{-3}$ range. This could affect the back contact as the native bulk doping level may not be sufficient for a good low resistance contact or to get appreciable majority carrier thermally assisted tunneling and thermionic emission over the back contact Schottky barrier.(9) The majority carrier current is likely to be further impeded by an increasing Schottky barrier energy with increasing Al-content, due to a decreasing CIAS valance band maximum energy.(10) This could be the reason for the high series resistance measured in the devices above. High-efficiency CIGS devices have order of magnitude higher doping levels than those measured on the CIAS devices herein, and high bandgap CGS devices do not show back contact related problems. This is presumably due to increasing net acceptor doping with increasing $\mathrm{Ga}$ content, and a valance band maximum energy that does not change much with increasing $\mathrm{Ga}$ content. These results lead us to believe that the high series resistance observed in the high Al-content CIAS devices is due in part to the back contact. Thus, the low doping densities in high Al-content CIAS could further complicate the ability to make low resistance transparent back contacts.

Several transparent conducting oxides (TCO'S) have been tested as IR transparent replacements to the standard opaque Mo back contacts on high- and low-bandgap CIGS devices. Indium Tin Oxide (ITO) was demonstrated to make good devices with low-bandgap CIGS (low Ga, $E_{g}=1.1 \mathrm{eV}$ ), while additionally showing good durability to the top cell deposition. However, high-bandgap CIGS or CIAS devices $\left(\mathrm{E}_{\mathrm{g}}>1.35 \mathrm{eV}\right)$ do not make good devices directly on ITO. Other TCO's such as $\mathrm{SnO}_{2}$ and $\mathrm{ZnO}$ did not fair well either, even with the low-bandgap CIGS, indicating that the type of TCO is important to the transparent back contact. Theoretically, this could be due to the decreasing valance band maximum with increasing bandgap, as previously discussed, and a lack of sufficient interface states or mid-gap states in the TCO for tunneling or thermally emitted holes followed by recombination processes. The energy band diagram of the back contact "Schottky" barrier at the p-type CIAS and degenerate ntype TCO interface, and the majority carrier tunneling and recombination process is depicted in Figure 6 below. These results suggest that an interface layer is necessary for wide-bandgap devices using TCO back contacts.
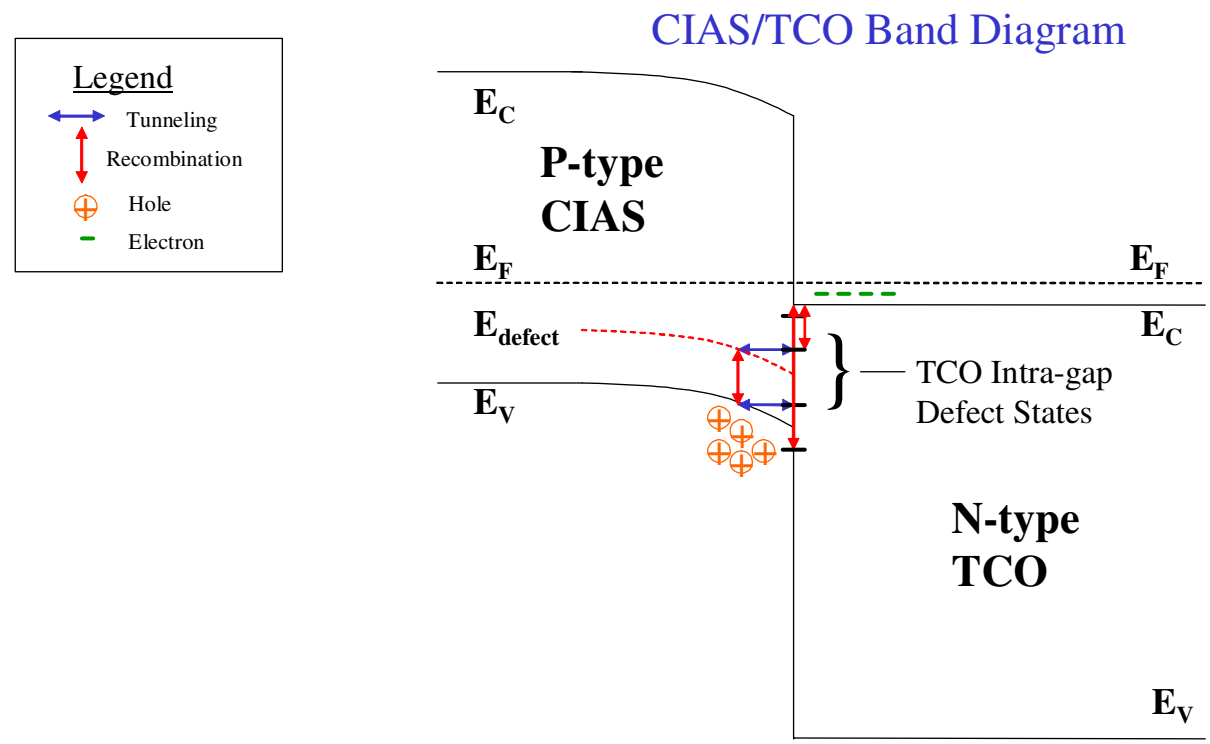

Figure 6 - Energy band diagram of the back contact "Schottky" barrier at the p-type CIAS and degenerate n-type TCO interface, and showing the majority carrier tunneling and recombination process. 
Since the earlier CIAS and CIGS device testing with TCO back contacts, ITN has developed an interface layer to the TCO's. The normalized transmission data for this interconnect is shown in Figure 7 below, and was tested to have an average transmission of about $71.5 \%$, weighted to the AMO solar irradiance in the range of $750 \mathrm{~nm}$ to $1300 \mathrm{~nm}$.

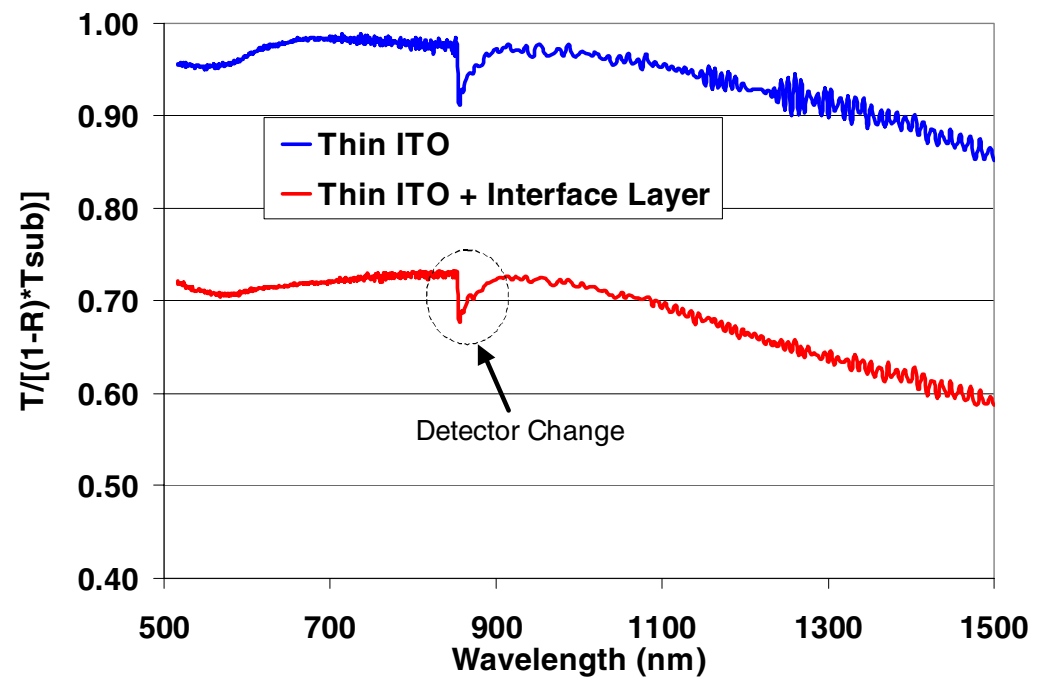

Figure 7 - Normalized Transmission (substrate divided out) of a thin ITO layer, and a thin ITO layer plus a top interface layer.

This level of transmission enables approximately $33 \%$ of the useable AMO solar power to be transmitted to the bottom cell in a tandem configuration when assuming a top cell bandgap of $1.65 \mathrm{eV}$ or $750 \mathrm{~nm}$, or to be transmitted out of the device for lower temperature operation from a wide-bandgap single junction device. In addition, this back contact would enable over $70 \%$ transmission of the visible spectrum to pass through, in the event of bifacial illumination.

The same semi-transparent back contact structure was used in the fabrication of CIAS devices (bandgap $\approx 1.35$ $\mathrm{eV}$ ) and efficiencies near $11 \%$ were obtained. These efficiencies were comparable to the performance of the controls using the standard opaque molybdenum back contacts. The results are shown in Table 2 below.

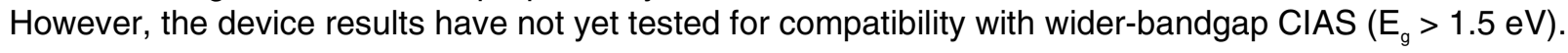

Table 2 - CIAS device with transparent back contact and Mo controls $(\mathrm{AI} /[\mathrm{Al}+\mathrm{In}]=0.35, \mathrm{Cu} /[\mathrm{Al}+\mathrm{In}]=0.69$, Tsub $\left.=450^{\circ} \mathrm{C}\right)$

\begin{tabular}{|c|c|c|c|c|}
\hline $\begin{array}{c}\text { Glass Substrate/ } \\
\text { Back Contact }\end{array}$ & $\begin{array}{c}\mathrm{V}_{\mathrm{oc}} \\
(\mathrm{Volts})\end{array}$ & $\begin{array}{c}\mathrm{J}_{\mathrm{sc}} \\
\left(\mathrm{mA} / \mathrm{cm}^{2}\right)\end{array}$ & $\begin{array}{c}\mathrm{FF} \\
(\%)\end{array}$ & $\begin{array}{c}\eta \\
(\%)\end{array}$ \\
\hline SL/Mo & 0.58 & 29.4 & 63.5 & 10.9 \\
7059/Mo & 0.56 & 28.8 & 65.7 & 10.7 \\
7059/Mo/TCO/Thin TIL* & 0.59 & 27.8 & 64.9 & 10.7 \\
\hline
\end{tabular}

* TIL - transparent interface layer

\section{SUMMARY}

Significant challenges exist in the development of high-efficiency wide-bandgap devices, but this development is still in its infancy and some possibilities for improvement exist. The wide-bandgap CIAS device performance 
without any bandgap tailoring to date has not yet proven to be any better than the CIGS performance at the same bandgap.(8) This equivalence supports some recent theories about deep defects becoming more effective by moving toward mid-gap with increasing bandgap.(11) However, there are enough differences between the two wide-bandgap CIS alloys to suggest the possibility of different mechanisms limiting their respective performance. These include differences in the intra-gap defect distribution, net native doping, and valance and conduction band maximum energies, all of which can have implications for device performance. The test results presented herein suggest that improvements to the CIAS near surface properties could improve the high Al content devices from the large-area, moving substrate deposition system. This could involve modified CIAS deposition near the surface or some post deposition surface treatments. The test results also suggest that a back contact interface layer may be needed to help improve the back contact. Other device test results with $\mathrm{CuGaSe}_{2}\left(\mathrm{E}_{g}=1.67 \mathrm{eV}\right)$ solar absorbers show dominant interface recombination, indicating that better matched alternative heterojunction partners or surface treatments may be needed for the wide-bandgap devices.(12)

Finally, the potential for obtaining good transparent back contacts to wide-bandgap devices is promising, with demonstrated IR transparency greater than $60 \%$ on some back contact structures and device efficiencies that are comparable to standard Mo back contacts, but there is still much room for improvement.

\section{ACKNOWLEDGEMENTS}

ITN would like to acknowledge the Institute of Energy Conversion for collaborative work performed on CIAS on TCO results. The results discussed herein were obtained by work funded by: NIST/Dept. of Commerce, Advanced Technical Program, Contract No. 70NANB8H4070; and NASA, Contract No.'s NAS3-02027 and NAS3-02170.

\section{REFERENCES}

\footnotetext{
${ }^{1}$ S.G. Bailey, A.F. Hepp, and R.P. Raffaelle, "Thin Film Photovoltaics for Space Applications," Proc. Of the $36^{\text {th }}$ IECEC, pp. 235-238, (2001).

${ }^{2}$ D. Hoffman, "A Parametric Assessment of the Mission Applicability of Thin-Film Solar Arrays," NASA Technical Memorandum: NASA/TM-2002-211720, (2002).

${ }^{3}$ K. Ramanathan, M.A. Contreras, C.L. Perkins, S. Asher, F. S. Hasoon, J. Keane, D. Young, M. Romero, W. Metzger, R. Noufi, J. Ward, A. Duda, "Properties of 19.2\% Efficiency CulnGaSe ${ }_{2}$ Thin-film Solar Cells", Progress in Photovoltaics: Research and Applications, Vol. 11, pp. 225-230, (2003).

${ }^{4}$ P. Paulson, M. Haimbodi, S. Marsillac, R. Birkmire and W. Shafarman, "Cu( $\left(\mathrm{n}_{1-x} A \mathrm{~A}_{x}\right) \mathrm{Se}_{2}$ Thin Films and Solar Cells, J. Appl. Phys., 91, pg. 10153, (2002).

${ }^{5}$ U. Rau, M. Schmidt, A. Jasenek, G. Hanna, H. Schock, "Electrical Characterization of Cu(In,Ga)Se ${ }_{2}$ Thin-Film solar Cells and the Role of Defects for the Device Performance," Sol. Energy Mat. \& Sol. Cells, 67, pp.137-143, (2001).

${ }^{6}$ J. Heath, J. Cohen, W. Shafarman, and D. Johnson, "Characterization of Deep Defects in Culn ${ }_{1-x} \mathrm{Ga}_{x} \mathrm{Se}_{2}(\mathrm{CIGS})$ Working Photovoltaic Devices," Photovoltaics for the $21^{\text {st }}$ Century II, The Electrochemical Society, Inc., New Jersey, pp. 324-332, (2001).

${ }^{7}$ S. Marsillac, P. Paulson, M. Haimbodi, R. Birkmire, and W. Shafarman, "High-Efficiency Solar Cells Based on $\mathrm{Cu}(\mathrm{InAl}) \mathrm{Se}_{2}$ Thin Films, Appl. Phys. Lett., 81,(7), pp. 1350-1352, (2002).

${ }^{8}$ W. Shafarman, S. Marsillac, P. Paulson, M. Haimbodi, T. Minemoto, and R. Birkmire, "Material and Device Characterization of Thin Film Cu(InAl)Se Solar Cells, Proc. of the $29^{\text {th }}$ IEEE PVSC, pp. 519-522, (2002).

${ }^{9}$ A. Fahrenbruch and R. Bube, "Fundamentals of Solar Cells - Photovoltaic Solar Energy Conversion," Academic Press, London, UK, 1983, pg. 169.

${ }^{10}$ S. B. Zhang, S.H. Wei, and A. Zunger, "A Phenomenological Model for Systematization and Prediction of Doping Limits in II-VI and I-III-VI, Compounds," J. Appl. Phys., 83(6), pp. 3192-3196, (1998).

${ }^{11}$ J. Heath, J. Cohen, W. Shafarman, D. Liao, and A. Rockett, "Effect of Ga Content on Defect States in Culn

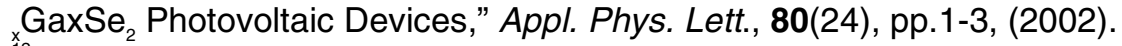

${ }_{12}$ J. Malmstrom, J. Wennerberg, M. Bodegard, and L. Stolt, "Influence of Ga on the Current Transport in $\mathrm{Cu}(\mathrm{In}, \mathrm{Ga}) \mathrm{Se}_{2}$ Thin Film Solar Cells," Proc. of the $17^{\text {th }}$ European Photovoltaic Solar Energy Conference and Exhibition, Munich, Germany, (2001).
} 